\title{
An Analysis of Higher Education Leadership in the United Arab Emirates
}

\section{Ramy Iskander}

American University of Ras Al Khaimah

\section{Lincoln Pettaway}

American University of Ras Al Khaimah

Lee Waller

American University of Ras Al Khaimah

\section{Sharon Waller}

American University of Ras Al Khaimah; Lee.Waller@aurak.ac.ae

Doi:10.5901/mjss.2016.v7n1p444

\section{Abstract}

\begin{abstract}
This study evaluated the effectiveness of a senior leader at an international institution of higher education in the United Arab Emirates. The questionnaire utilized in the study was developed by an organizational taskforce employing the frameworks of servant leadership. The project solicited perceptions from all employees regarding (1) leadership focus, (2) strategic insight and ability, (3) building effective teams and community, along with (4) ethical formation and decision-making. A quantitative methodology was utilized for this study. The paper conducted a factor analysis for dimension reduction on the findings of the questionnaire. Results were analyzed for reliability and validity. The instrument was found to be highly reliable with a Chronbach alpha of 0.977 . The evaluation of validity identified only one underlying factor driving the organizational responses. This factor was identified as the employee's global perception. The study evidences a holistic view of leadership rather than a component view.
\end{abstract}

Keywords: Leadership, Servant-Leadership, United Arab Emirates, Higher Education

\section{Introduction}

Ras Al Khaimah (RAK) is the fastest growing and most northern emirate in the U.A.E. Over the past ten years, RAK has become a destination for many tourist and investors. Numerous light and medium manufacturing firms, as well as, service industries are located throughout RAK. Additionally, many educational institutions are located within and around the emirate. Numerous educational entities operate in the RAK Free Trade Zone and do not comply with the accreditation requirements imposed on those institutions operating outside the Free Trade Zone (Alhebsi, Pettaway, \& Waller, 2015). This study examined the leadership effectiveness of a senior administrator (chief executive officer) of an educational entity operating outside the RAK Free Trade Zone and under the accreditation expectations of the Commission of Academic Accreditation (CAA), Ministry of Higher Education and Scientific Research, United Arab Emirates.

The accreditation standards of the CAA (2011, Standard 1.3.7, p. 7) clearly articulate the role of the Chief Executive Officer (CEO). The CEO has the task of ensuring that the institution accomplishes the vision and mission adopted by the Board. The Board delineates institutional policy while the CEO handles the day-to-day operation of the institution. The accreditation standards of the CAA (2011, Standard 1.4.7, p. 7) also clearly articulate the expectation for organizational effectiveness to include the annual evaluation of the effectiveness of the CEO in leading the organization. The evaluation may or may not include a grassroots evaluation of the performance of the CEO. The approach utilized in the evaluation is left to the decision of the Board. 


\section{Research Design}

In compliance with the specified accreditation expectations of the CAA, the institution examined in the study moved to develop a meaningful, grassroots evaluation of the leadership practices of the Chief Executive Officer. A taskforce was established and charged with the creation of an evaluation instrument. The taskforce developed the organizational questionnaire under the frameworks of servant leadership as espoused by Greenleaf (1970). The taskforce identified four major areas for examination: (1) leadership focus, (2) strategic insight and ability, (3) building effective teams and community, and (4) ethical formation and decision-making. Questions for each major area were developed and implemented. Responses were collected, tabulated and evaluated.

\subsection{Research Question}

This study examined the responses of the institution's personnel to answer the following research question. Do relationships exist between or among the responses of Ras Al Khaimah personnel related to the evaluation of the effectiveness of senior leadership?

\subsection{Research Hypotheses}

The following null and alternate hypotheses were utilized to guide this study and support the analysis required by the study's research question.

Ho: No relationships exist between or among the responses of Ras Al Khaimah personnel related to the evaluation of the effectiveness of senior leadership.

Ha: Relationships exist between or among the responses of Ras Al Khaimah personnel related to the evaluation of the effectiveness of senior leadership.

\subsection{Research Methodology}

The quantitative research method utilized in this study made use of a Chronbach alpha to evaluate reliability and factor analysis to determine if relationships existed between or among the responses of Ras Al Khaimah personnel relating to the effectiveness of the institution's senior leadership. Waller and Lumadue (2013) state that factor analysis provides the researcher with the opportunity to answer questions that pertain to the relationship of variables being studied. Factor analysis was utilized to examine the relationships between all variables within this study. The factor analysis employed data reduction techniques to identify underlying latent variables that are reflected in the observed variables. Factors with initial Eigenvalues above 1.0 were considered significant.

Dunteman (1989) established four assumptions for factor analysis. The four assumptions follow.

1. The data sets must not contain outliers.

2. The sample size must be adequate and the case must be greater than the factor.

3. The model must be linear in nature.

4. Interval data are assumed.

The researcher examined each of these assumptions to provide the informed consumer appropriate information by which to assess the findings of the study. The data included no outliers and were linear in nature. The adequacy of sampling size was assessed using the Kaiser-Meyer-Olkin measure of sampling adequacy. The adequacy of sampling size was satisfied with a score of 0.924 . Reliability of the survey responses was examined utilizing the Chronbach's alpha. Chronbach's alpha is a measure of scale reliability (Institute for Digital Research and Education, 2015). A reliability coefficient of 0.70 or higher is deemed to be acceptable (Lumadue \& Waller, 2013). The study evidenced a Chronbach alpha of 0.977 . The usefulness of the research is left to the determination of the informed reader.

\subsection{Limitations and Delimitations}

This study was limited by the following factors.

1. Data included in the study were self-reported by individual personnel. As such, all the traditional limitations of self-reported data apply.

2. The data were available only for the fall 2015 semester. No data were available prior to this time.

The researcher delimited the study as follows. 
1. The study was restricted to data retrieved utilizing the survey instrument.

2. The collection of information was delimited to full-time employees only.

3. The research examined only the parameters identified by the taskforce.

\subsection{Assumptions}

The following assumptions were made for this study.

1. The data were accurate and correctly recorded.

2. The data were usable and appropriate for this study.

3. Examination of the data has the potential to impact future practice and research.

\section{Findings and Analysis}

The research question called for an analysis of the relationship between or among the responses to the 20-item questionnaire. Ninety-two (92) responses were obtained. The Bartlett's Test of Sphericity was utilized to determine if any relationship existed between or among the responses. The significance of the Bartlett's Test of Sphericity was found to be less than 0.001 . This finding indicated the presence of relationships within the responses. Accordingly, the null hypothesis, $\mathrm{H}_{0}$, was rejected in favor of the alternate hypothesis, $\mathrm{H}_{\mathrm{a}}$. Relationships were found to exist between or among the responses of Ras Al Khaimah personnel related to the evaluation of the effectiveness of senior leadership. Factor analysis was next conducted to identify the relationships between the various responses. Findings of the factor analysis are included in Table 1.

Table 1. Total Variance Explained

\begin{tabular}{ccccccc}
\hline & \multicolumn{3}{c}{ Initial Eigenvalues } & \multicolumn{3}{c}{ Extraction Sums of Squared Loadings } \\
\cline { 2 - 6 } Component & Total & \% of Variance & Cumulative \% & Total & \% of Variance & Cumulative \% \\
\hline 1 & 14.109 & 70.543 & 70.543 & 14.109 & 70.543 & 70.543 \\
Extraction Method: Principal Component Analysis & & & &
\end{tabular}

Table 1 provides the total variance explained by the model. Only one factor was identified with an eigenvalue above 1.0. This factor explained $70.543 \%$ of the variance and can be viewed as a general perception of the leadership style of the administrator evaluated. The scree plot is provided in Figure 1. The scree plot demonstrates that one major factor is determining the majority of the variance. The remaining factors are not sufficient to account for the remaining variance. Accordingly, only one major factor underlies the responses to the questionnaire. This implies that the questionnaire only measures one driving factor. This factor can be explained as a global or holistic perception of the leadership style of the administrator being evaluated. Leadership is viewed as only on major issue rather than the compilation of various components or perceptions of components.

As evidenced in Table 2 which follows, every factor loading for the identified factor held almost equal weight with factor loading scores ranging from a low of 0.756 to a high of 0.908 . Accordingly, the factor loadings were deemed to be very homogenous in their impact on the identified factor. One might argue that the holistic perception of the senior administrator's leadership led to almost equal responses on the various questions. This occurred in spite of the efforts of the taskforce to ensure that the questionnaire was founded on 4 major components of Greenleaf's servant leadership model. 


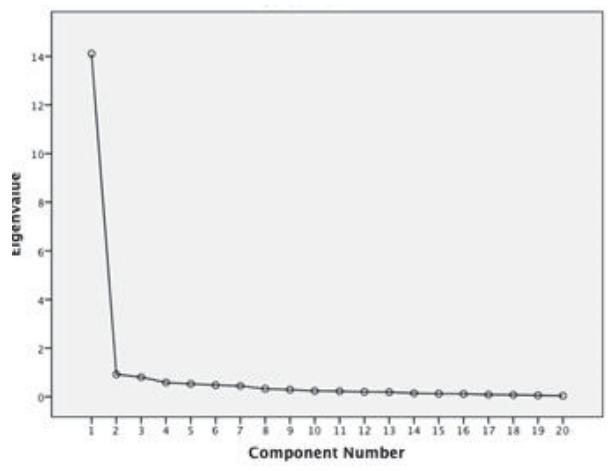

Figure 1. Scree Plot

The component matrix for each of the identified factors is provided in Table 2. As previously noted, only one factor had sufficient impact on the explained variance. The remaining factors do not significantly explain the variance.

Table 2. Component Matrix

\begin{tabular}{lc}
\hline Component & Factor Loading \\
\hline 1. Leadership Focus - High Expectations & 0.835 \\
2. Leadership Focus - Inspires Others & 0.881 \\
3. Leadership Focus - Maximizes Opportunity & 0.890 \\
4. Leadership Focus - Assumes Responsibility & 0.868 \\
5. Leadership Focus - Promotes Success & 0.829 \\
6. Strategic Insight - Communicates Mission & 0.756 \\
7. Strategic Insight - Aligns Plans & 0.856 \\
8. Strategic Insight - Quick to Adapt & 0.836 \\
9. Strategic Insight - Communicates Ramifications & 0.803 \\
10. Strategic Insight - Accepts Full Responsibility & 0.806 \\
11. Teaming - Shared Responsibility & 0.772 \\
12. Teaming - Treats People Fairly & 0.855 \\
13. Teaming - Solicits Best Possible Solution & 0.790 \\
14. Teaming - Establishes Culture of Engagement & 0.891 \\
15. Teaming - Respectful of Divergent Opinions & 0.834 \\
16. Ethics - Professional Behavior & 0.873 \\
17. Ethics - Applies Policies Fairly & 0.808 \\
18. Ethics - Appreciation and Sensitivity & 0.908 \\
19. Ethics - Does His/Her Share of Task & 0.850 \\
20. Ethics - Demonstrates Loyalty to Institution & 0.838 \\
\hline
\end{tabular}

Extraction Method: Principal Component Analysis

\section{Conclusions and Implications}

An examination of the analysis revealed the existence of a relationship between the responses to the various questions relating to the CEO's leadership of the institution and the holistic perception of the CEO's leadership ability. The single factor associated with this holistic perception of the individual's leadership ability explained most of the variance in spite of efforts on the part of the taskforce to develop a questionnaire relating to four unique component areas associated with Greenleaf's servant leadership model. The study's findings indicated that the questionnaire only obtained one general perception of the CEO's leadership ability.

Generalizability of these findings is limited. The case study considered 92 responses to an assessment of effectiveness for one CEO in one institution in Ras Al Khaimah, UAE. However, the findings indicate that the leadership ability was perceived holistically rather than by components. Though caution should be taken in generalizing the conclusions beyond the study, one issue is clear. Leadership can be and is often evaluated based on a single 
phenomenon or perception rather than by the examination of individual leadership components. Leaders should carefully consider the powerful impact of holistic perception of an individual's leadership ability on the many components of leadership styles and strategies. Recognition of the impact of perceiving leadership as a holistic phenomenon can serve to guide a senior administrator in carefully weighing all actions capable of shaping an employee's holistic perception of how well an institution is being led. Simply put: the holistic perception of quality leadership shapes the perception of the individual component areas associated with leadership. Care must be taken to maintain the holistic perception that the institution is being effectively led as a means of empowering perception of the attributes and component areas composing a leadership strategy. When the holistic perception suffers, the perception of other leadership components is likely to suffer. When the holistic perception is empowered, the perception of other leadership components is also likely to be empowered. When it comes to leadership, the whole impacts the parts.

\section{References}

Alhebsi, A., Pettaway, L., \& Waller, L. (2015). A history of education in the United Arab Emirates and Trucial Shiekdoms, The Global eLearning Journal, 4(1), Retrieved from https://globalelearningjournal.files.wordpress.com/2010/11/a-history-of-education-in-theunited-arab-emirates-and-trucial-sheikdoms.pdf

Commission for Academic Accreditation. (2011). Standards for Licensure and Accreditation, Ministry of Higher Education and Research, United Arab Emirates, retrieved August 3, 2015 from https://www.caa.ae/caa/images/Standards2011.pdf

Dunteman, G. H. (1989). Principal Components Analysis. Newbury Park, CA: Sage.

Greenleaf, R. (1970). The Servant as Leader: Essays. Atlanta, GA: Robert Greenleaf Center for Servant Leadership.

Institute for Digital Research and Education (2015). What does the Cronbach's alpha mean? Retrieved May 8, 2015 from http://www.ats.ucla.edu/stat/spss/fag/alpha.html.

Lumadue, R. \& Waller, L. (2013). Educational Research Today. (1st ed.). Cupertino, CA: Apple iTunes Connect. Retrieved from https://itunes.apple.com/us/book/educational-research-today/id691632696?mt=11

Waller, L., \& Lumadue, R. (2013). Factor Analysis. (1st ed.). Cupertino, CA: Apple iTunes Connect. Retrieved from https:/litunes.apple. com/us/book/factor-analysis/id656956844?ls=1 